\title{
Improved Field Homogeneity for Transmission Line MRI Coils Using Series Capacitors
}

\author{
Zhurbenko, Vitaliy; Dong, Yunfeng
}

Published in:

Proceedings. 2015 SBMO/IEEE MTT-S International Microwave and Optoelectronics Conference (IMOC)

Link to article, DOI:

10.1109/IMOC.2015.7369186

Publication date:

2015

Document Version

Peer reviewed version

Link back to DTU Orbit

Citation $(A P A)$ :

Zhurbenko, V., \& Dong, Y. (2015). Improved Field Homogeneity for Transmission Line MRI Coils Using Series Capacitors. In Proceedings. 2015 SBMO/IEEE MTT-S International Microwave and Optoelectronics Conference (IMOC) IEEE. https://doi.org/10.1109/IMOC.2015.7369186

\section{General rights}

Copyright and moral rights for the publications made accessible in the public portal are retained by the authors and/or other copyright owners and it is a condition of accessing publications that users recognise and abide by the legal requirements associated with these rights.

- Users may download and print one copy of any publication from the public portal for the purpose of private study or research.

- You may not further distribute the material or use it for any profit-making activity or commercial gain

- You may freely distribute the URL identifying the publication in the public portal 


\title{
Improved Field Homogeneity for Transmission Line MRI Coils Using Series Capacitors
}

\author{
Vitaliy Zhurbenko and Yunfeng Dong \\ Technical University of Denmark \\ Kgs. Lyngby, Denmark \\ vz@elektro.dtu.dk
}

\begin{abstract}
High field magnetic resonance imaging (MRI) systems often use short sections of transmission lines for generating and sensing alternating magnetic fields. Due to distributed nature of transmission lines, the generated field is inhomogeneous. This work investigates the application of series capacitors to improve the field homogeneity. The resulting magnetic field distribution is estimated analytically and evaluated numerically. The results are compared to a case of a conventional transmission line coil realization.
\end{abstract}

Keywords-magnetic resonance imaging; MRI coil; transmission line; TEM coil

\section{INTRODUCTION}

The essential part of any magnetic resonance (MR) system is radiofrequency (RF) coils. The purpose of RF coils is to generate and sense alternating magnetic fields in the imaging domain of the system. While loop coils is a conventional choice for low field (low frequency) MR applications [1], [2], transmission lines operating in standing wave mode are often used as coils in high field systems. The maxima and minima of the standing wave on the line results in inhomogeneous magnetic field distribution. Such inhomogeneity leads to degradation of MR image quality.

Several approaches to improve homogeneity have been suggested in the literature. These include implementation of alternating impedance transmission lines [3], [4], metamaterial based structures [5], [6] , and substituting the coil with several shorter coils with individual feeding [7], [8]. In this work, the approach of inserting series capacitors into transmission line coil is investigated. Series capacitors compensate for phase shift generated by the equivalent series inductor of the transmission line, which results in a more uniform current distribution on the line. The uniform current distribution, in its turn, leads to homogeneous magnetic field. The equations for optimal values of series capacitors are presented. The achieved homogeneity of the magnetic field is estimated using Biot-Savart's law and compared to a conventional transmission line coil. An example of a transmission line coil using microstrip technology is considered.

\section{CoIl Design AND CURREnT Distribution}

\section{A. Current Distribution on a Transmission Line}

For higher efficiency, the transmission line coils are always operated as resonators. Hence, the loading of the transmission line is either open circuit, short circuit, or purely reactive. Such a loading results in infinite standing wave ratio (assuming lossless case) where the distance between the consecutive minima or maxima is one-half a wavelength [9]. To avoid dark spots on MR image and achieve reasonable homogeneity, current minima should be avoided. For that reason, coils based on transmission lines are always shorter than half a wavelength and proper capacitive loading $\left(C_{\mathrm{L}}\right)$ is used to achieve symmetric current distribution along the line. An example of such a setup and corresponding magnitude of the current distribution $|I(z)|$ are shown in Fig. 1.

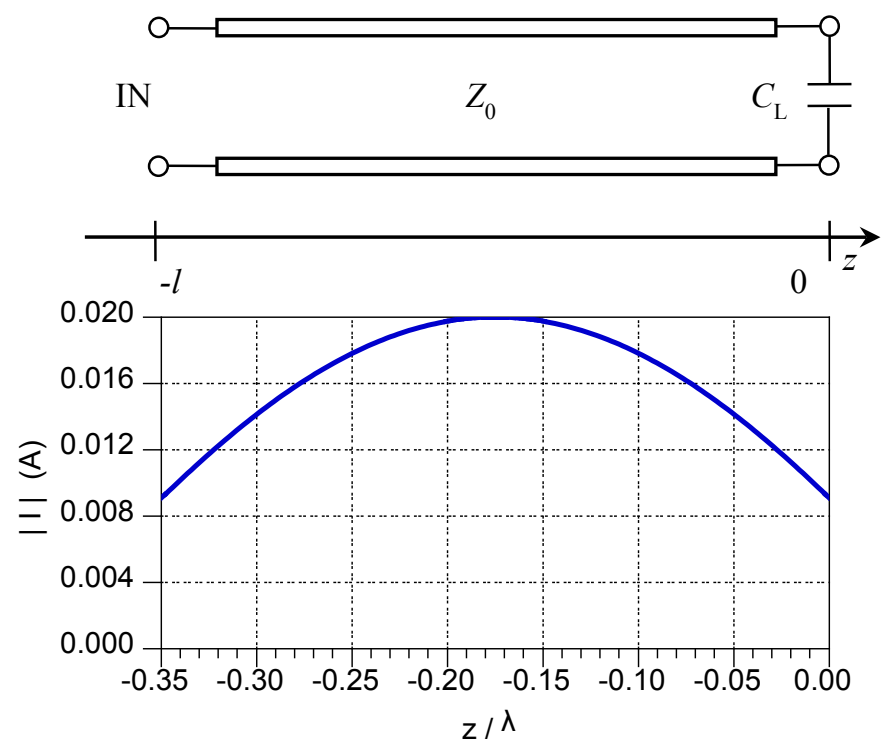

Fig. 1. Above - capacitively loaded transmission line coil; below corresponding magnitude of a current distribution assuming the line is connected to a $1 \mathrm{~V}$ generator having impedance of $50 \Omega$. 
The value of the capacitor is usually chosen such that the maximum of the current magnitude appears right in the middle of the transmission line section. The current magnitude reduces moving towards the ends of the line due to destructive interference of the incident and reflected waves.

Ideally, it would be preferable to have a uniform current distribution. That would result in homogeneous magnetic field along the line (assuming that the line is infinitely long).

For practical reasons the size of the coils (length of the transmission line section) is usually limited to cover a certain field for view. A line length of $0.35 \cdot \lambda$ (where $\lambda$ is a guided wavelength) was chosen as an example here, but in general, the considered here approach is applicable to coils which are longer that one-half of a wavelength.

\section{B. Coil with Series Capacitors}

A uniform current distribution can be achieved by inserting series capacitors into the transmission line. An equivalent circuit of a short lossless transmission line section consists of a series inductor and parallel capacitor as shown in Fig. 2. These elements shift the phase of propagating waves, which then results in destructive interaction of incident and reflected waves in certain places on the line.

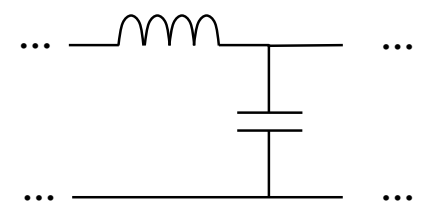

Fig. 2. Equivalent circuit for a short section of a lossless transmission line.

Using series capacitors, as shown in Fig. 3. can partially compensate for that, by introducing opposite phase shift.

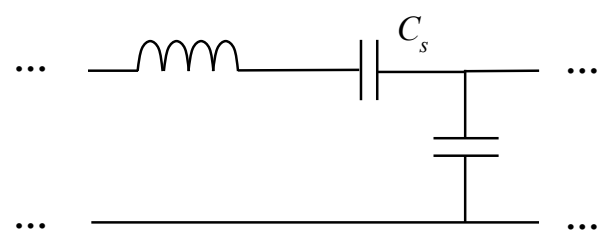

Fig. 3. Equivalent circuit for a short section of a lossless transmission line with compensating series capacitor.

Implementing this capacitor will give a smaller standing wave ratio than in the conventional case in Fig. 1. An example of using just one series capacitor is shown in Fig. 4 (black dashed line). As can be seen in Fig. 4, the resulting uniformity of the current distribution is considerably improved.

Obviously, the higher the number of capacitors, the more uniform current distribution can be achieved, and consequently, the higher the magnetic field homogeneity will be. An example of using nine capacitors inserted between ten equal length transmission line sections is also shown in Fig. 4.

The expression for the loading capacitor, which will result in such an optimal current distribution is derived imposing current symmetry condition for $l / N$ length line [10]:

$$
C_{L}=\frac{-\sin (\beta l / N)}{2 \pi f_{0} z_{0}(\cos (\beta l / N)-1)},
$$

where $l$ is the total length of the transmission line coil; $N$ is the number of transmission line sections after inserting series capacitors (for example, using one series capacitor will result in $N=2) ; \beta$ is the phase constant; $Z_{0}$ is the characteristic impedance of the implemented transmission line; $f_{0}$ is the operating frequency.
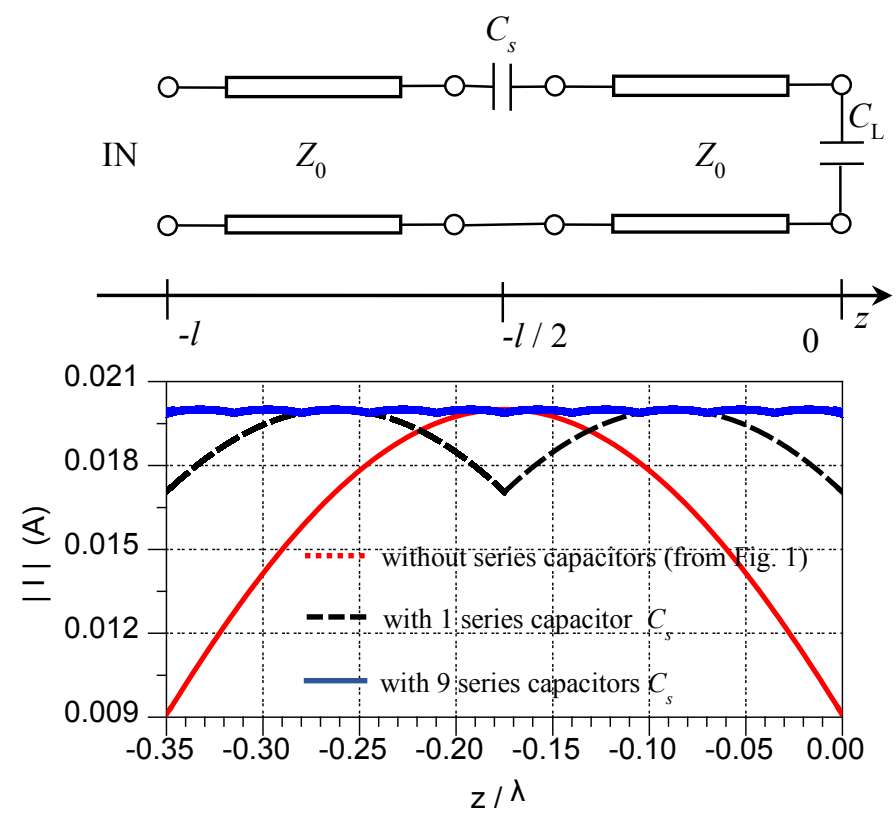

Fig. 4. Above - capacitively loaded transmission line coil with one series capacitor; below - corresponding magnitude of the current distribution and comparison to the conventioal line case and multicapacitor case. It is assumed, that the line is connected to a $1 \mathrm{~V}$ generator having impedance of $50 \Omega$.

The value of the series capacitor $C_{s}$ should be chosen such that it compensates the phase shift introduced by a section of the transmission line:

$$
C_{s}=C_{L} / 2
$$

Having the values of the capacitors and knowing the parameters of the transmission line, current distribution can be found using well-developed transmission line analysis:

$I(z)=$

$$
=\left\{\begin{array}{cl}
0 & \text { when } 0<z<-N \cdot d l \\
I_{n}(z) & \text { when }-(N-n-1) d l \leq z \leq-(N-n) d l
\end{array}\right.
$$

where $I_{n}(z)$ is the current on $n^{\text {th }}$ transmission line section, $n=1 \ldots N$, and $d l=l / N$ is the length of one transmission line 
section. A schematic representation of the considered transmission line coil with multiple series capacitors is shown in Fig. 5.

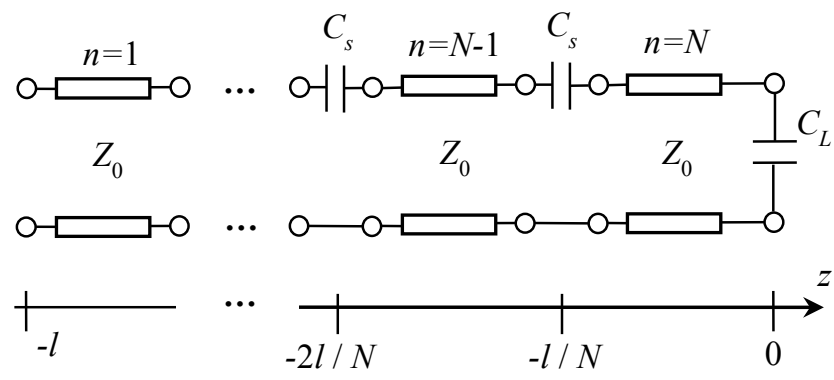

Fig. 5. Transmission line coil with multiple series capacitors.

$$
I_{n}(z)=\frac{V_{0, n}^{+}}{Z_{0}}\left(e^{-j \beta(z+(N-n) d l)}-\Gamma_{L, n} e^{j \beta(z+(N-n) d l)}\right)
$$

The amplitude of the incident wave at the end of $n^{\text {th }}$ transmission line section

$$
V_{0, n}^{+}= \begin{cases}\frac{V_{g}}{2} \frac{\left(1-\Gamma_{g}\right) e^{-j \beta d l}}{1-\Gamma_{g} \Gamma_{L, n} e^{-j 2 \beta d l}} & \text { when } n=1 \\ \frac{V_{i n, n}}{e^{j \beta d l}+\Gamma_{L, n} e^{-j \beta d l}} & \text { when } 2 \leq n \leq N\end{cases}
$$

Here $V_{g}$ is the voltage of the generator, which excites the transmission line coil, $V_{i n, n}$ is the total voltage at the input terminals of $n^{\text {th }}$ transmission line section. Generator reflection coefficient $\Gamma_{g}=\frac{Z_{g}-Z_{0}}{Z_{g}+Z_{0}}$, where $Z_{g}$ is the impedance of the generator. Reflection coefficient at the end of $n^{\text {th }}$ transmission line section $\Gamma_{L, n}=\frac{Z_{L, n}-Z_{0}}{Z_{L, n}+Z_{0}}$, where

$$
\begin{aligned}
& Z_{L, n}= \\
& = \begin{cases}\frac{1}{j 2 \pi f_{0} C_{L}} & \text { when } n=N \\
\frac{1}{j 2 \pi f_{0} C_{s}}+Z_{0} \frac{Z_{L, n+1}+j Z_{0} \tan (\beta d l)}{Z_{0}+j Z_{L, n+1} \tan (\beta d l)} & \text { when } 1 \leq n \leq N-1\end{cases}
\end{aligned}
$$

The field strength due to the current distribution $I(z)$ can be estimated using Biot-Savart law:

$$
|\mathbf{H}(z, r)|=\frac{r}{4 \pi} \int_{-l}^{0} \frac{\left|I\left(z^{\prime}\right)\right| d z^{\prime}}{\left(r^{2}+\left[z^{\prime}-z\right]^{2}\right)^{3 / 2}}
$$

where $r$ is the perpendicular distance to the conductor carrying the current from the point of observation. Since the magnetic flux density is proportional to the magnetic field $(\mathbf{B}=\mu \mathbf{H}$, where $\mu$ is the permeability), it is possible to estimate the produced $\mathbf{B}$ field [8]. Integration in (4) is limited to $-l \ldots 0$ since there is no current outside this region (refer to eq. (3)).

\section{MAGNETIC FIELD HomogeneITy}

Equation (4) is used to estimate the field distribution due to current in Fig. 1 and for the case with nine series capacitors (Fig. 5) having $N=10$ (corresponding current distribution is shown in Fig. 4). The results for a range of $r$ from $0.03 \lambda$ to $0.12 \lambda$ is shown in Fig. 6.

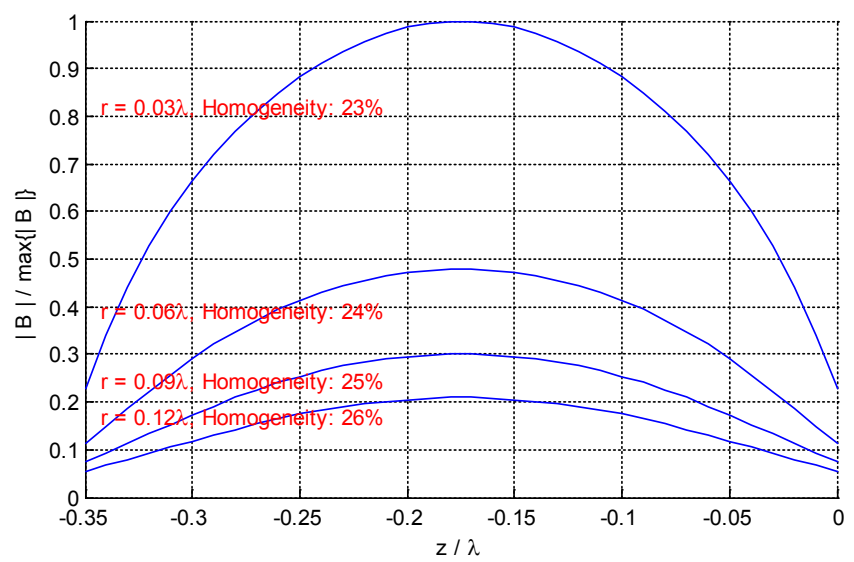

(a)

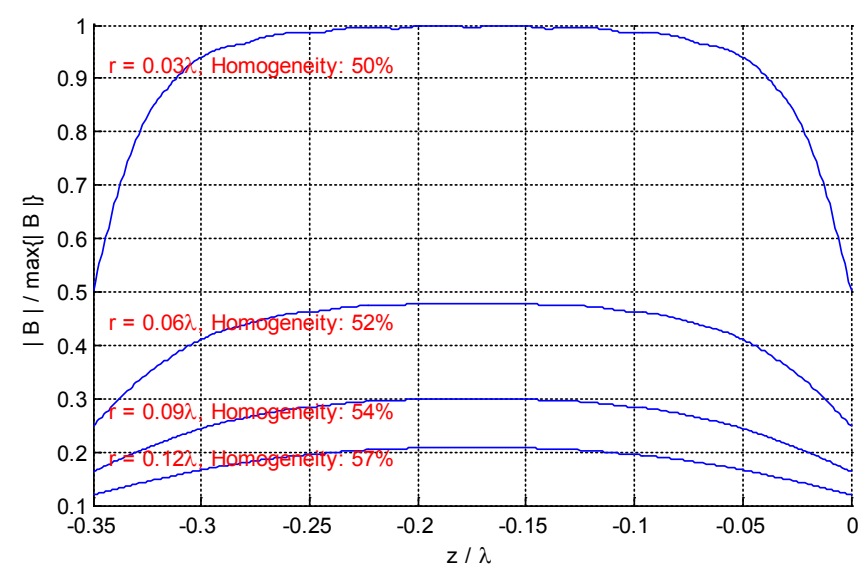

(b)

Fig. 6. Normalized magnitude of $\mathbf{B}$ field distribution (a) due to current in Fig. 1, (b) for the case with nine series capacitors.

The presented data indicates, that the insertion of series capacitors considerably improves homogeneity of the field. For example for $r=0.03 \lambda$ homogeneity, which is defined as a ratio

$$
\min \{|\mathbf{B}(\mathrm{z})|\} / \max \{|\mathbf{B}(\mathrm{z})|\},
$$

increases from $23 \%$ to $50 \%$. For $50 \Omega$ line the calculated using eq. (1), (2) values of the capacitors at $f_{0}=300 \mathrm{MHz}$ and $N=10$ are $C_{L} \approx 96.1 \mathrm{pF}$ and $C_{s} \approx 48.05 \mathrm{pF}$. 
As it can also be seen from Fig. 6, reducing the field of view (the imaging domain above the transmission line) increases homogeneity of the field. For instance, if the field of view is reduced to $0.25 \lambda$ (keeping the same coil length $0.35 \lambda$ ), the achieved field homogeneity will increase from $66 \%$ for the conventional transmission line coil to almost $94 \%$ for the coil with compensating series capacitors.

\section{Microstrip Line COIL}

The considerations above are, in general, applicable to any coil based on TEM transmission line. To confirm the results obtained in the previous section, a more specific example of a transmission line coil using microstrip technology is considered here. The impedance of the implemented microstrip transmission line is $50 \Omega$. The height (distance between the ground plane and the strip) is $5 \mathrm{~mm}$, and the width of the line is $24.4 \mathrm{~mm}$. The substrate relative permittivity is 1 providing pure TEM mode (there is, however, no reason to believe that quasiTEM mode microstrip lines would not exhibit a similar behavior). The total length of the coil is $35 \mathrm{~cm}$, which at $f_{0}=300 \mathrm{MHz}$ corresponds to electrical length of approximately $0.35 \lambda$. The chosen frequency is close to operating frequency of MR systems for hydrogen imaging with 7T magnetic field.

The pattern of the microstrip lines with overlaid magnitude of longitudinal component of the surface currents are shown in Fig. 7.

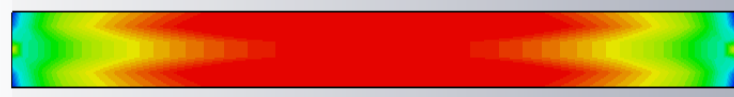

(a)

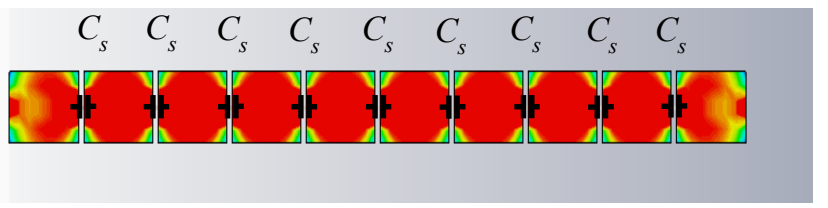

(b)

Fig. 7. Magnitude of longitudial component of the surface current on the cunductor of (a) a conventional microstrip line versus (b) line with nine series capacitors.

The shown current distributions are obtained by full-wave electromagnetic simulations.

As expected, the current distribution on the microstrip line (Fig. 7(a)) resembles the distribution for the ideal transmission line shown in Fig. 1 with maximum amplitude at the center and minimum at the ends. As predicted by the analysis in the previous Section, the current distribution on the microstrip line with series capacitors is more uniform (Fig. 7(b)). It can also be seen in Fig. 7(b) how the natural current flow gets disturbed by forcing most of the current to flow through the lumped series capacitor in the middle of the line, while, conventionally, the current in microstrip lines is mostly concentrated at the edges of the line. This contributes to a current component which is transversal to the direction of the wave propagation. To diminish this effect, one could either use two capacitors positioned at the edges of the microstrip line instead of one capacitor in the middle, or use a distributed series capacitor [10].

It should be mentioned, that the microstrip structure has parasitic fringing capacitance at the ends of the transmission line sections and current in the loading capacitor. To compensate for that and non-ideal lumped port excitation, the value of the loading capacitor of the conventional transmission line coil (Fig. 7 (a)) has been reduced to $4.81 \mathrm{pF}$ (the initial value calculated from eq. (1) is $5.41 \mathrm{pF}$ ). The corresponding value for the microstrip line with nine series capacitors (Fig. 7(b)) $C_{L}=67.1 \mathrm{pF} . C_{s}=33.55 \mathrm{pF}$. After this adjustment the relationship $C_{s}=C_{L} / 2$ still holds providing symmetric current distribution.

The field distribution obtained by full-wave simulation for the same range of $r$ is shown in Fig. 8 .

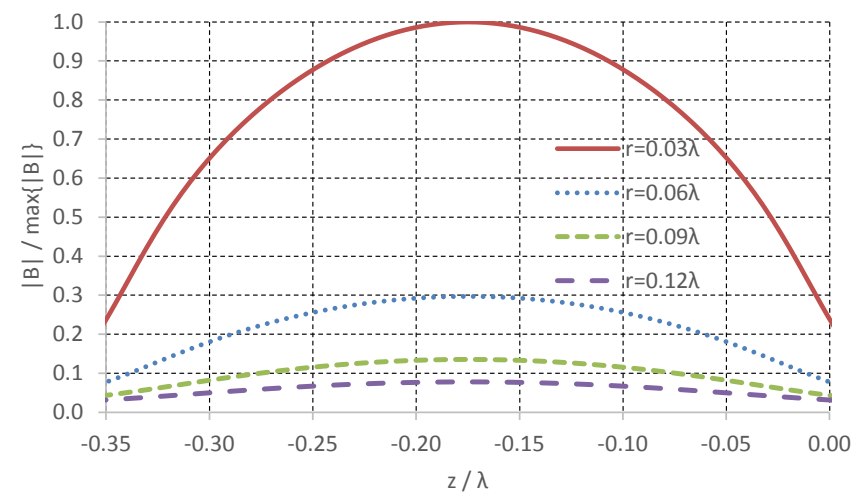

(a)

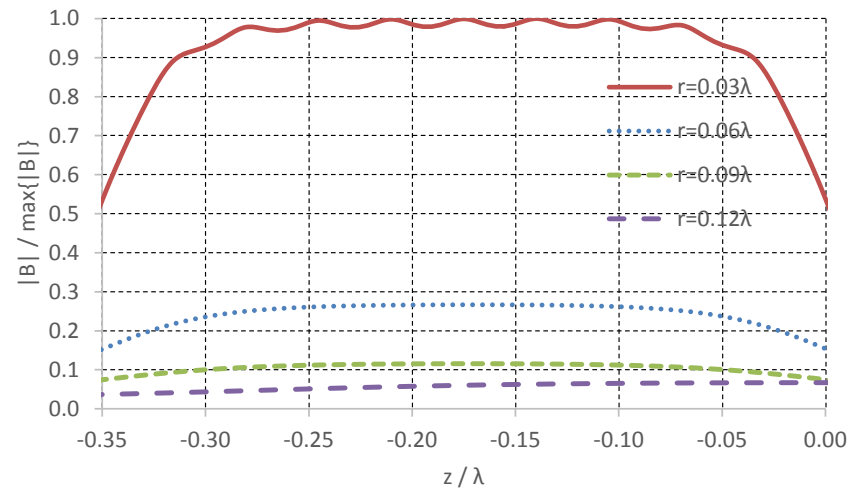

(b)

Fig. 8. Normalized magnitude of the $\mathbf{B}$ field distribution (a) for the conventional microstrip transmission line coil, (b) for the capacitively loaded microstrip transmission line coil with nine series capacitors.

The presented data confirms, that the insertion of series capacitors considerably improves homogeneity of the field to approximately the same degree, as it is predicted using simplified analysis. For example, for $r=0.03 \lambda$ homogeneity increases from $23 \%$ to $53 \%$.

The absolute simulated values of the fields are, however, lower than the values predicted because the current on the ground plane has an opposite direction and generates the field counteracting the field generated by the current on the strip. For 
that reason the ratio between the field values for different $r$ in Fig. 6 and Fig. 8 are not exactly the same.

\section{CONCLUSION}

It was demonstrated, that the magnetic field homogeneity for the transmission line MR coils can be considerably improved by inserting series capacitors. The higher the number of series capacitors, the higher the homogeneity is achieved. In practice, however, the number of the capacitors should be limited to avoid possible degradation of the transmission line resonator quality factor due to eventual parasitic losses in capacitors. An attractive alternative would be to use distributed series capacitors. The equations for optimal values of capacitors are given.

It is shown, that the profile of the current distribution can be changed inserting series capacitors. Evenly distributed capacitors have been investigated in this work (equal value capacitors inserted between equal length line sections). Even higher homogeneity over a wider field of view could be achieved using unevenly distributed capacitors with unequal values.

The design approach can be used to construct transmission line coils which are longer than one-half of a wavelength.

\section{ACKNOWLEDGMENT}

The authors would like to thank Danish National Research Foundation (grant DNRF124) for partial support of the activities.

\section{REFERENCES}

[1] RF Coils for MRI, edited by J. T. Vaughan, and J. R. Griffiths, A John Wiley and Sons, Ltd., 2012, p.474.

[2] D. Nilsson, J. J. Mohr, and V. Zhurbenko, "Practical Aspects of 13C Surface Receive Coils with Active Decoupling and Tuning Circuit," Proceedings of the 42nd European Microwave Conference: 29 October 1 November 2012, Amsterdam, The Netherlands, pp. 65-68.

[3] I. A. Elabyad, A. Omar, "An Investigation of Alternating Impedance Microstrip Transceiver Coil Arrays for MRI at 7T," Proceedings of the International Microwave Symposium, 5 - 10 June 2011, Baltimore, pp. 1-4.

[4] C. E. Akgun, L. DelaBarre, H. Yoo, S.-M. Sohn, C. J. Snyder, G. Adriany, K. Ugurbil, A. Gopinath, and J. T. Vaughan, "Stepped Impedance Resonators for High-Field Magnetic Resonance Imaging," IEEE Transactions on Biomedical Engineering, Vol. 61, No. 2, February 2014, pp.327-333.

[5] A. Rennings, J. Mosig, A. Bahr, C. Caloz, M. E. Ladd, D. Erni, "A CRLH Metamaterial based RF Coil Element for Magnetic Resonance Imaging at 7 Tesla," Proceedings of 3rd European Conference on Antennas and Propagation, EuCAP 2009, pp. 3231 - 3234.

[6] A. Senn, A. Peter, and J. G. Korvink, "An 8-channel metamaterial T-R coil at 9.4T," Proceedings of ISMRM Annual Meeting, 2011, Montréal, Québec, Canada, p.1

[7] X. Yan, J. O. Pedersen, L. Wei, X. Zhang, R. Xue, "Multi-channel doublerow transmission line array for human MR imaging at ultrahigh fields," IEEE Trans Biomed Eng., Volume: 62, Issue: 6, June 2015, pp. 1652 1659.

[8] J. O. Pedersen, "Simulation and Fabrication of 16-Element Double-Row ICE Decoupled Microstrip Coil," Master Thesis, Sino-Danish Center for Education and Research, 2014.

[9] P. Meincke and J. Vidkjær "Introduction to Wireless RF System Design," Lecture Notes.

[10] V. Zhurbenko "Optimal Value of Series Capacitors for Uniform Field Distribution in Transmission Line MRI Probes". 